\title{
Early Reperfusion Rates with IV tPA Are Determined by CTA Clot Characteristics
}

\author{
S.M. Mishra, J. Dykeman, T.T. Sajobi, A. Trivedi, M. Almekhlafi, S.I. Sohn, S. Bal, E. Qazi, A. Calleja, M. Eesa, M. Goyal,
}

A.M. Demchuk, and B.K. Menon

\begin{abstract}
BACKGROUND AND PURPOSE: An ability to predict early reperfusion with IV tPA in patients with acute ischemic stroke and intracranial clots can help clinicians decide if additional intra-arterial therapy is needed or not. We explored the association between novel clot characteristics on baseline CTA and early reperfusion with IV tPA in patients with acute ischemic stroke by using classification and regression tree analysis.
\end{abstract}

MATERIALS AND METHODS: Data are from patients with acute ischemic stroke and proximal anterior circulation occlusions from the Calgary CTA data base (2003-2012) and the Keimyung Stroke Registry (2005-2009). Patients receiving IV tPA followed by intra-arterial therapy were included. Clot location, length, residual flow within the clot, ratio of contrast Hounsfield units pre- and postclot, and the M1 segment origin to the proximal clot interface distance were assessed on baseline CTA. Early reperfusion (TICI 2a and above) with IV tPA was assessed on the first angiogram.

RESULTS: Two hundred twenty-eight patients ( $50.4 \%$ men; median age, 69 years; median baseline NIHSS score, 17) fulfilled the inclusion criteria. Median symptom onset to IV tPA time was 120 minutes (interquartile range $=70$ minutes); median IV tPA to first angiography time was 70.5 minutes (interquartile range $=62$ minutes). Patients with residual flow within the clot were 5 times more likely to reperfuse than those without it. Patients with residual flow and a shorter clot length $(\leq 15 \mathrm{~mm}$ ) were most likely to reperfuse $(70.6 \%)$. Patients with clots in the M1 MCA without residual flow reperfused more if clots were distal and had a clot interface ratio in Hounsfield units of $<2$ (36.8\%). Patients with proximal $\mathrm{Ml}$ clots without residual flow reperfused $8 \%$ of the time. Carotid-T/-L occlusions rarely reperfused (1.7\%). Interrater reliability for these clot characteristics was good.

CONCLUSIONS: Our study shows that clot characteristics on CTA help physicians estimate a range of early reperfusion rates with IV tPA.

ABBREVIATIONS: CART = classification and regression tree; cirHU = clot interface ratio in Hounsfield units; IA = intra-arterial; $I Q R=$ interquartile range; $T C D=$ transcranial Doppler

A cute ischemic stroke treatment is primarily focused on dissolving clots within the arterial tree by using thrombolytic agents administered intravenously or by endovascular tech-

Received January 19, 2014; accepted after revision May 6

From the Departments of Clinical Neurosciences (S.M.M., T.T.S., A.T., M.A., E.Q., M.E., M.G., A.M.D., B.K.M.), Radiology (J.D., M.A., M.E., M.G., A.M.D., B.K.M.), and Community Health Sciences (T.T.S., B.K.M.), University of Calgary, Calgary, Alberta, Canada; Department of Neurology (S.I.S.), Dongsan Medical Center, Keimyung University, Daegu, South Korea; Faculty of Medicine (M.A.), King Abdulaziz University, Jeddah, Saudi Arabia; Department of Neurology (S.B.), University of Manitoba, Winnipeg, Manitoba, Canada; Department of Neurology (A.C.), Hospital Clinico Universitario, University of Valladolid, Valladolid, Spain; Seaman Family MR Center (M.A., E.Q., M.E., M.G., A.M.D., B.K.M.), Calgary, Alberta, Canada; and Hotchkiss Brain Institute (T.T.S., M.G., A.M.D., B.K.M.), Calgary, Alberta, Canada.

Paper previously presented at: International Stoke Conference, February 11-14, 2014; San Diego, California.

Please address correspondence to Bijoy K. Menon, MD, Department of Clinical Neurosciences and Radiology, University of Calgary, Hotchkiss Brain Institute, Seaman Family MR Center, Foothills Medical Centre, 1403 29th St NW, Calgary, Alberta,

Canada T2N2T9; e-mail: docbijoymenon@gmail.com

http://dx.doi.org/10.3174/ajnr.A4048 niques. Since the approval of IV tPA for thrombolysis, effort has been made to identify clot characteristics on imaging that predict recanalization with IV tPA. The "hyperattenuated" sign on NCCT is a marker of intracranial clot. ${ }^{1-3}$ Its location (MCA versus Sylvian) plays an important role in determining clinical outcome. ${ }^{4-8}$ In addition, the length of the hyperattenuated segment on NCCT, especially with ultrathin-section nonenhanced CT reconstructions, correlates with recanalization and good clinical outcome. ${ }^{9,10}$ Clot characteristics on CTA that predict recanalization include location and burden. ${ }^{1-13}$ Residual flow on transcranial Doppler (TCD) is an imaging marker of recanalizing clot. ${ }^{14,15}$

Current evidence supports the role of early reperfusion in improving clinical outcome in patients with acute ischemic stroke. ${ }^{16,17}$ If clinicians are able to estimate early reperfusion rates with IV tPA, they can make decisions favoring rescue intra-arterial (IA) therapy in patients with a low likelihood of early reperfusion with IV tPA. 


\begin{tabular}{|c|c|c|c|}
\hline & $\begin{array}{l}\text { Calgary CTA Data Base } \\
\qquad(n=165)\end{array}$ & $\begin{array}{l}\text { Keimyung Stroke } \\
\text { Registry }(n=63)\end{array}$ & $P$ Value \\
\hline Age (yr) (mean) & $66.4 \pm 14.3$ & $69.5 \pm 9.96$ & .12 \\
\hline Sex (male) (\%) & $49.1 \%$ & $53.9 \%$ & .5 \\
\hline Baseline NIHSS (median) (IQR) & $18(8)$ & $14(6)$ & .01 \\
\hline Onset to IV tPA time (minutes) (median) (IQR) & $112(73)$ & $125(57)$ & .18 \\
\hline IV tPA initiation to first angiography run time (minutes) (median) (IQR) & $53(45)$ & $139(75)$ & .01 \\
\hline \multicolumn{4}{|l|}{ Site of occlusion (No.) (\%) } \\
\hline Carotid-T/-L & $13(8 \%)$ & $1(1.6 \%)$ & .09 \\
\hline Tandem occlusions & $38(23 \%)$ & $23(36.5 \%)$ & \\
\hline Ml MCA & $86(52.1 \%)$ & $31(49.2 \%)$ & \\
\hline M2 MCA & $28(17 \%)$ & $8(12.7 \%)$ & \\
\hline $\begin{array}{l}\text { Very early arterial-weighted CTA (preclot } \mathrm{HU}<150 \text { and preclot } \\
\qquad \mathrm{HU} \geq \mathrm{HU} \text { at the torcula) (No.) (\%) }\end{array}$ & $0(0 \%)$ & $2(3.2 \%)$ & .07 \\
\hline $\begin{array}{l}\text { Very late venous phase CTA (preclot } \mathrm{HU}<150 \text { and preclot } \mathrm{HU}<\mathrm{HU} \\
\text { at the torcula) (No.) (\%) }\end{array}$ & $1(0.6 \%)$ & $2(3.2 \%)$ & .19 \\
\hline Residual flow (\%) & $21.8 \%$ & $4.8 \%$ & .01 \\
\hline Clot length (mm) (median) (IQR) & $22.3(34.5)$ & $50(30.6)$ & .01 \\
\hline cirHU (median) (IQR) & $1.5(0.85)$ & $2.8(1.5)$ & .01 \\
\hline $\begin{array}{l}\text { M1 MCA origin to proximal clot interface distance }(\mathrm{mm}) \text { (median) } \\
\text { (IQR) }\end{array}$ & $10.6(7.6)$ & $9.6(11.3)$ & .74 \\
\hline
\end{tabular}

In this study, we report early reperfusion rates with IV tPA stratified by several clot characteristics measured on CTA. Using classification and regression tree analysis (CART), we then derived an algorithm to help clinicians estimate early reperfusion rates with IV tPA in patients with proximal clots. ${ }^{18}$

\section{MATERIALS AND METHODS}

Data are from patients presenting with acute ischemic stroke and proximal anterior circulation occlusions from the Calgary CTA data base (2003-2012) and the Keimyung Stroke Registry (20052009). Details of both registries have been described in previous publications. ${ }^{10,19}$ Similarities and differences in baseline characteristics of patients in these registries are described in Table 1.

Data from both registries were combined for the present analysis to have a sufficient sample size for robust statistical analyses and to increase the generalizability of results. All patients underwent an NCCT of the head at admission followed by CTA of the head and neck. Only patients who received IV tPA followed by a conventional cerebral angiography (DSA) for IA therapy were included in our study. Information on demographic and clinical characteristics was collected at baseline. Stroke severity was assessed by using the NIHSS at baseline, at discharge, and at 90 days. Functional status was assessed by using the mRS at similar timepoints. Interval times from stroke-symptom onset to presentation in the emergency department, imaging, thrombolysis, and endovascular procedures were also collected. The local ethics boards approved both studies.

\section{Image Acquisition and Analysis}

Standard nonhelical NCCT scanning was performed on a multisection scanner with a 5-mm section thickness. NCCT was followed by CTA with a helical scan technique. Coverage was from the arch to the vertex with continuous axial sections parallel to the orbitomeatal line of 0.625 - to $1.25-\mathrm{mm}$ section thickness. Acquisitions were obtained after a single bolus intravenous contrast injection of $70-120 \mathrm{~mL}$ of nonionic contrast media into an antecubital vein at 3-5 mL/s, autotriggered by the appearance of con- trast in a region of interest manually placed in the ascending aorta. Differences in protocol between the centers included the following: 1) the use of a scanner (HD75; GE Healthcare, Milwaukee, Wisconsin) versus a Somatom Sensation scanner (Siemens, Erlangen, Germany), and 2) CTA acquisition autotriggered by the appearance of contrast in the arch of the aorta versus the common carotid artery. Patients were taken to the angiography suite for revascularization after receiving IV tPA. The first angiogram of the ipsilesional arterial tree on DSA was used to identify early reperfusion with IV tPA. Baseline and follow-up imaging was analyzed at the imaging core lab of the Calgary Stroke Program. OsiriX, Version 4 (http://www.osirix-viewer.com) was used to reconstruct 2D multiplanar reconstruction images in axial, coronal, and sagittal planes by using 24-mm-thick slabs. An independent reader (M.A.) assessed reperfusion on the first DSA run and final angiography. ${ }^{17}$

\section{Clot Characteristics}

The following clot characteristics on baseline CTA were studied while the reader was blinded to conventional angiogram and clinical data.

Clot Location. Clot location was divided into 4 groups: carotidT/-L, tandem (cervical ICA and M1 MCA occlusions with a patent intracranial ICA), M1 MCA, and proximal M2 MCA occlusions. M1 MCA was defined as a vessel extending from the ICA bifurcation to the origin of the first major branch in the Sylvian sulcus.

Clot Length. Clot length was measured on CTA by using 3-mm multiplanar reconstructions in the axial plane (Fig 1). Whenever the proximal or distal end of clot could not be identified, clot length was imputed to $50 \mathrm{~mm}$. This imputation happened most frequently with ICA clots in which the proximal end was in the neck. We chose $50 \mathrm{~mm}$ as our imputation value because no clots that were measurable had lengths of $>50 \mathrm{~mm}$. Our use of nonparametric statistics ensures that this imputation does not affect results. 
Residual Flow within the Clot. Residual flow within the clot has been described in the TCD literature but never before by using CTA. ${ }^{14,15}$ If we saw clearly visibly increased contrast attenuation through the clot compared with surrounding brain parenchyma, it was classified as the presence of residual flow (Fig 2). Clots that
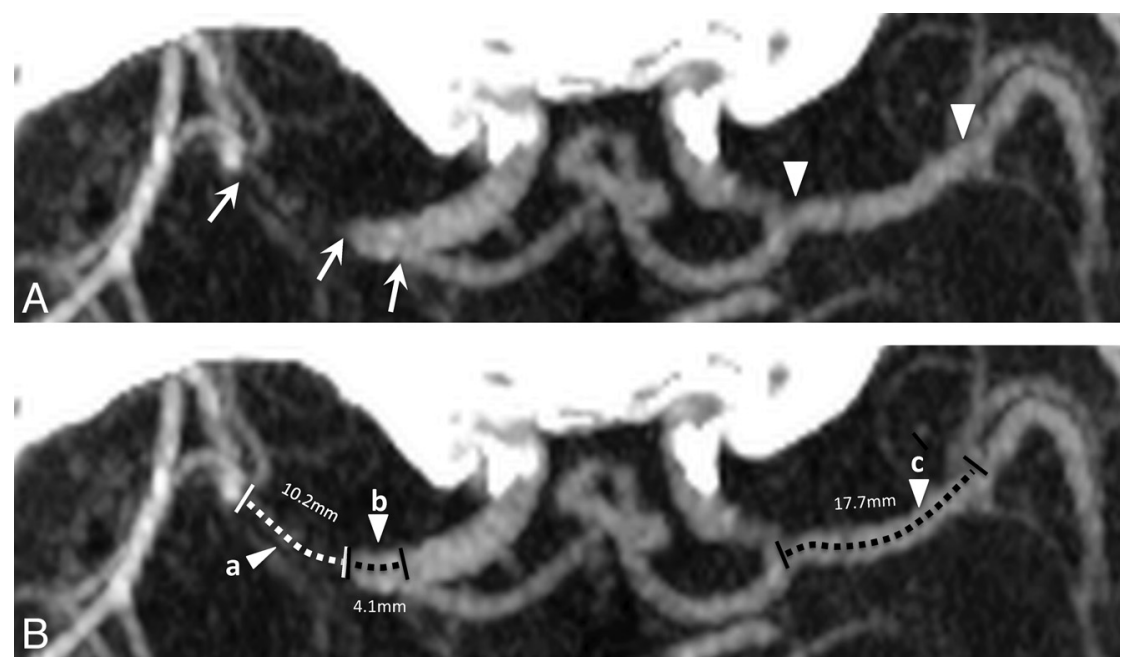

FIG 1. A, M1 MCA occluded segment (white arrows) with patent proximal M1 MCA on the right and the contralateral MI MCA (white arrowheads). B, Clot length (broken white line; segment a) and distance from M1 MCA origin to proximal clot interface (broken black line; segment b). Measurement of the contralateral Ml MCA segment is shown for reference (segment c). are hyperattenuated on NCCT could have increased signal attenuation on CTA; we found no correlation between the presence of the hyperattenuated sign on NCCT and what we classified as residual flow on CTA source images (Fisher exact test; $P$ value $=$ .76), thus suggesting that residual flow on CTA is not due to hyperattenuated clots on NCCT. We then graded residual flow within clot as follows - grade 0: clot with no contrast permeation and attenuation similar to that in surrounding brain parenchyma; grade I: clot appearing denser than surrounding brain parenchyma, with contrast potentially permeating through the clot; grade II: hairline or streak of well-defined contrast across the partial or complete length of the clot. Patients with intravascular nonocclusive thrombus on baseline CTA were considered as having early reperfusion at baseline and therefore excluded from analyses.

Clot Interface Ratio in Hounsfield Units. Residual flow within the clot may not always be visible to the naked eye due to partial volume effects on CTA. If, however, contrast signals at the proximal and distal clot

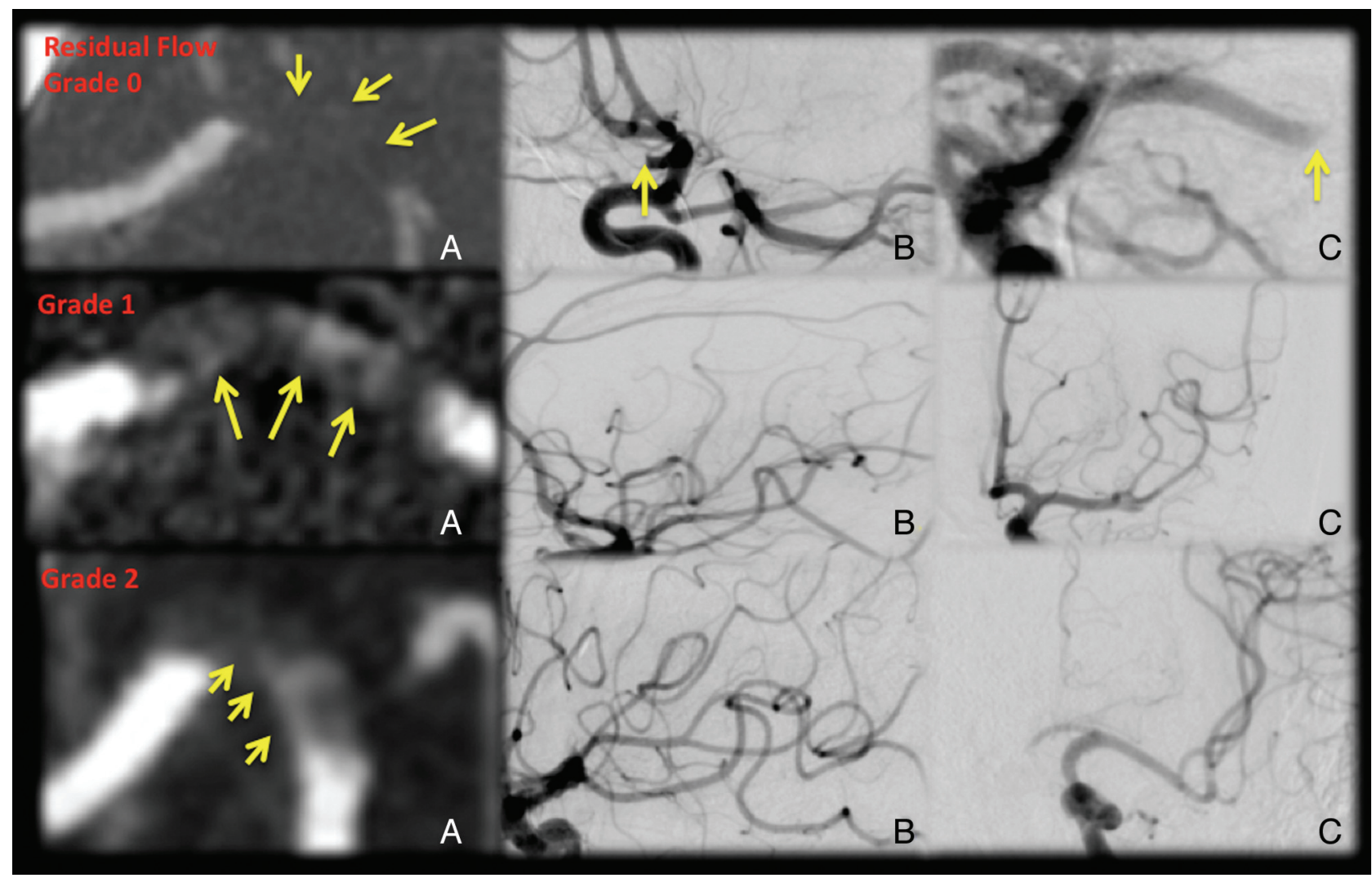

FIG 2. Residual flow on baseline CT angiography along with early reperfusion with IV TPA assessed on the first angiogram of the ipsilesional arterial tree. The top panel shows a patient with a left M1 MCA clot and no residual flow (A, grade 0 residual flow, yellow arrows, density similar to that of surrounding brain parenchyma). The first angiogram shows no recanalization ( $B$ and $C$ ). The middle panel shows a left M1 MCA clot with grade 1 residual flow ( $A$, yellow arrows, denser than surrounding brain parenchyma). The first angiogram shows excellent reperfusion ( $B$ and $C$ ). The bottom panel shows a left M1 MCA clot with grade 2 residual flow (A, yellow arrows, hairline or streak of well-defined contrast across the partial or complete length of the clot). The first angiogram shows excellent reperfusion ( $B$ and $C)$. 

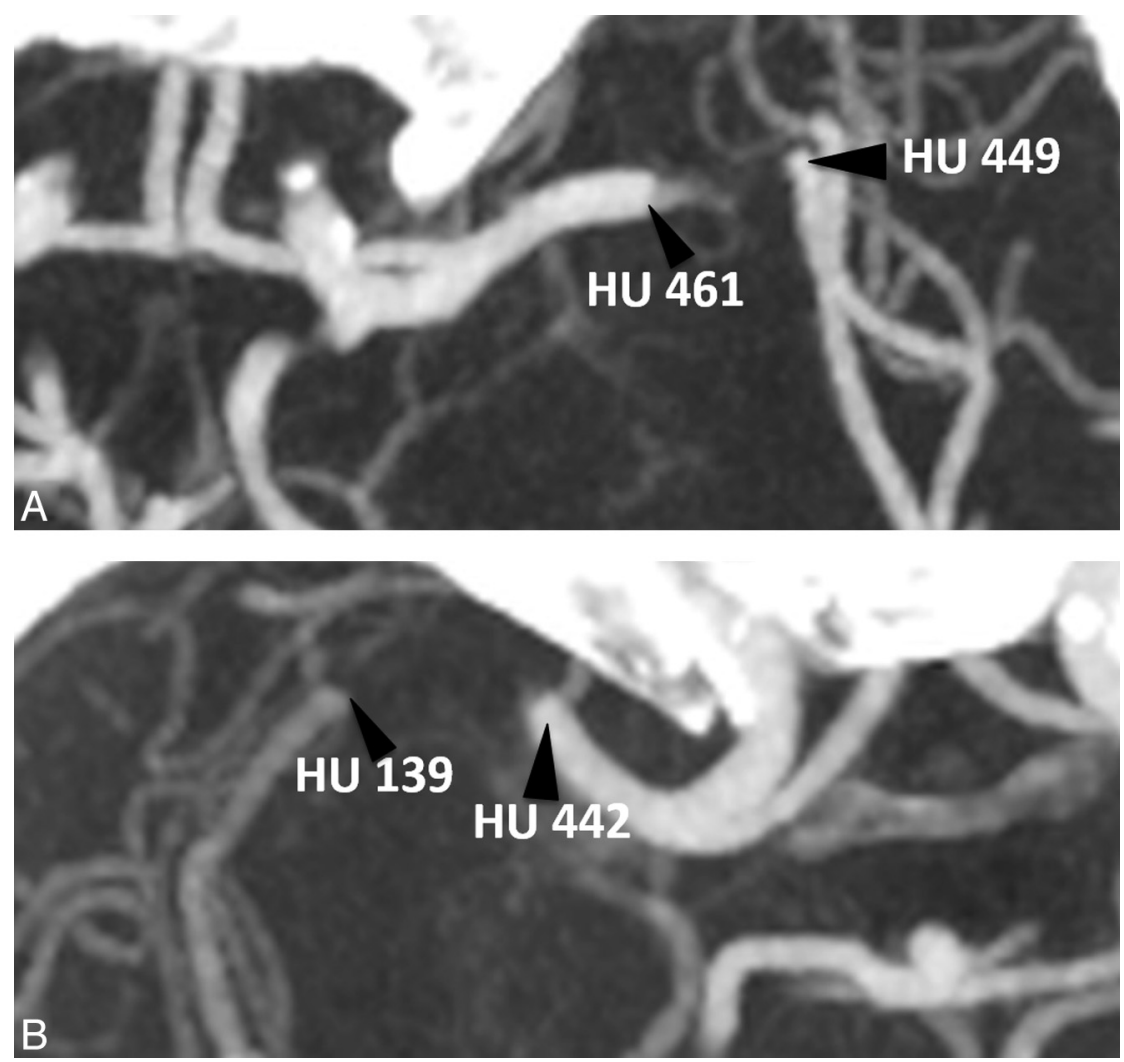

FIG 3. Clot interface Hounsfield unit ratio calculated by measuring the Hounsfield units in a region of interest selected at the proximal and distal clot interface only in scans that are mid- to late arterial- or appropriate venous-weighted. cirHU is calculated by dividing the proximal clot interface Hounsfield unit by the distal clot interface Hounsfield unit. In $A$, a patient with a left MI MCA clot has a cirHU of 1.05 while in B, a patient with a left M1 MCA clot has a cirHU of 3.21.

\section{Early Reperfusion}

All past and current literature reports recanalization/reperfusion with IV tPA by using either TCD Thrombolysis in Brain Infarction grades or Thrombolysis in Myocardial Infarction grades. ${ }^{11,14,15} \mathrm{We}$, therefore, chose to use TICI $2 \mathrm{a} / 2 \mathrm{~b} / 3$ on the first angiogram of the ipsilesional arterial tree on DSA as our primary measure of reperfusion; this grade is comparable with Thrombolysis in Myocardial Infarction 2 and will therefore give readers an ability to compare the rates we report with those in previous literature. Nonetheless, we also reported reperfusion rates measured as TICI $2 \mathrm{~b} / 3$ as a secondary outcome measure and performed sensitivity analyses with this latter outcome.

\section{Statistical Analyses}

Categoric data are reported by using proportions, ordinal data, and continuous data, with a skewed distribution by using medians and continuous data with a normal distribution by using means. Because the proximal or distal end of the clot was not identified in some patients, an arbitrary value of $>50 \mathrm{~mm}$ was assigned to such patients. Our choice of nonparametric statistics based on ranks for this variable ensured that this imputation did not affect results. Association between clot

interface (in an appropriate venous-weighted scan) are similar, the similarity is a potential marker of residual flow through the clot or of good collateral status. If contrast signal at the proximal end of the clot is high but drops at the distal end, it could mean no residual flow and/or poor collateral status. The presence of residual flow and/or good collaterals could be associated with increased reperfusion rates with IV tPA. To test this hypothesis, we calculated the clot interface ratio in Hounsfield units (cirHU) by dividing the proximal clot interface Hounsfield units by the distal clot interface Hounsfield units (Fig 3). CirHU was not calculated whenever proximal or distal clot interface Hounsfield units could not be measured. We decided to exclude from analyses CTAs that were very early arterial weighted (preclot Hounsfield units $<150$ and greater than Hounsfield units at the torcula) because this would affect the validity of our assumptions on cirHU being a marker of residual flow and/or good collaterals.

Distance from the M1 MCA Origin to the Proximal Clot Interface. For M1 MCA occlusions, we studied an additional clot characteristic by measuring the distance from the M1 MCA origin to the proximal clot interface. ${ }^{20,21}$ Distal M1 MCA clots may be exposed to more shear stress at the proximal clot interface due to patent flow in the lenticulostriate arteries compared with proximal clots. Additionally, distal M1 clots are potentially smaller than proximal clots. Smaller clots with more shear stress at the clot interface may lyse more with IV tPA..$^{20,22}$ characteristics and early reperfusion (TICI 2a to 3 ) was studied by using the Fisher exact test of proportions for categoric data and the Wilcoxon rank sum test for nonparametric data. In addition, we did 3 sensitivity analyses: 1) analyses restricted to M1 MCA occlusions with reperfusion defined as TICI $2 \mathrm{a}-3$; and 2) analyses of the whole sample with reperfusion defined as TICI $2 b / 3$; and finally 3 ) similar analyses restricted to each of the 2 populations (ie, the Calgary and the Keimyung data) in our sample. A 2-sided $\alpha<.05$ was considered statistically significant. These analyses were performed by using STATA/SE 12.1 software (StataCorp, College Station, Texas).

We then used a recursive partitioning classification and a regression tree (CART, Fig 4) to model the relationship between early reperfusion and various clot characteristics. CART is a distribution-free regression method that builds a tree by recursively partitioning the data into increasingly homogeneous subgroups to maximize the explained variance within each subgroup. At each stage (node), the CART algorithm selects the explanatory variable and splitting value that gives the best discrimination between 2 outcome classes. A full CART algorithm adds nodes until they are homogeneous or contain few observations. Our use of CART as a model-building strategy helped us explore the rich nonlinear interactions between various clot characteristics, determine collinearity, and predict early reperfusion while deriving estimates of reperfusion. ${ }^{18}$ Predictor variables included resid- 

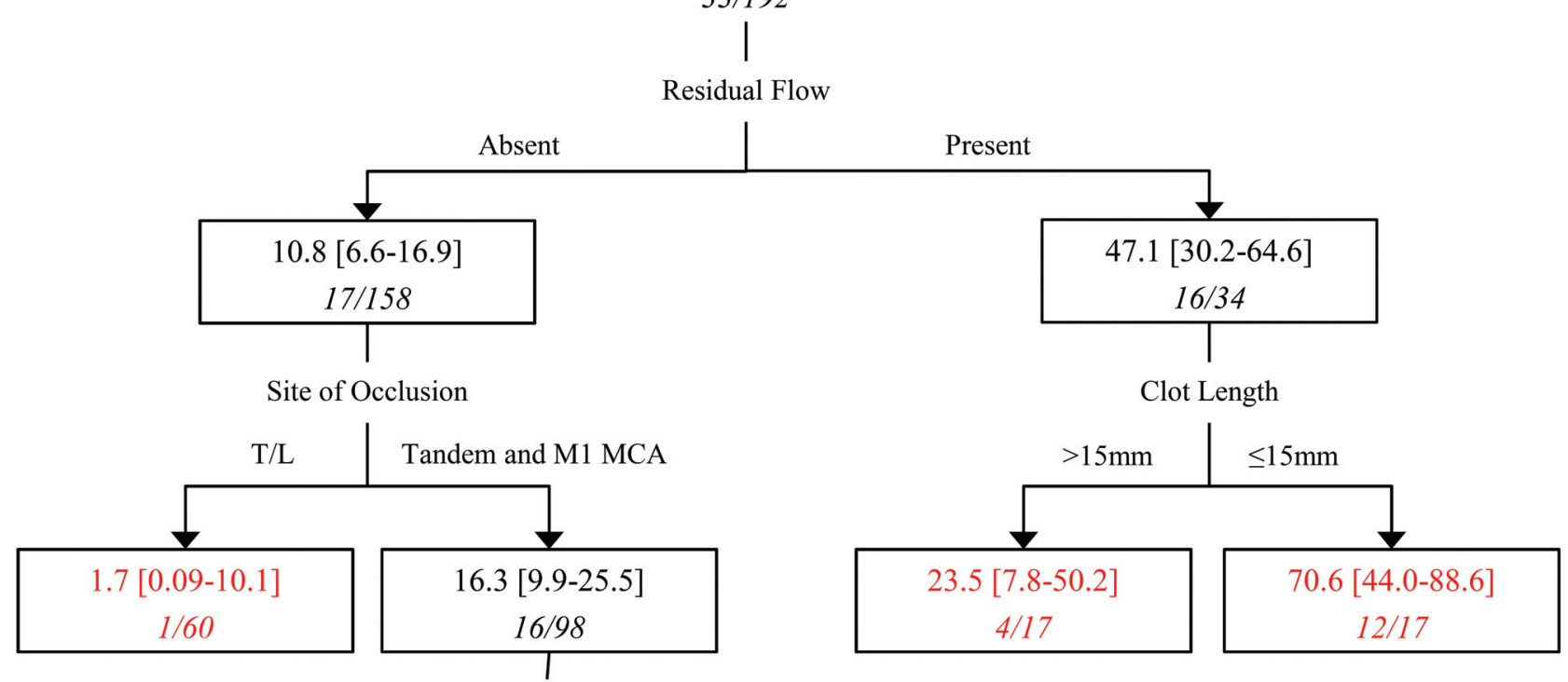

Distance from M1 origin to proximal Clot

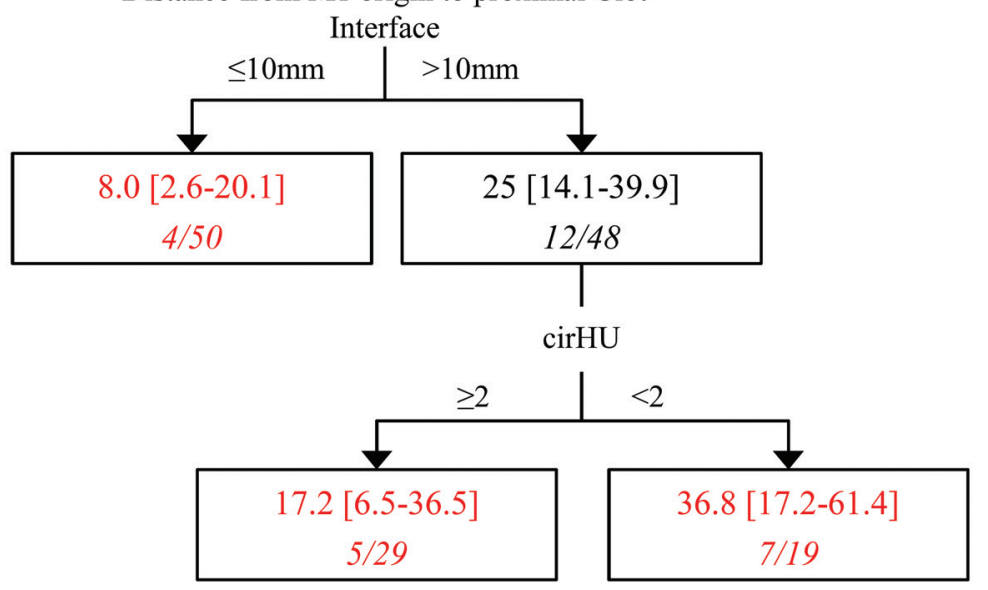

FIG 4. Tree representation of a recursive partitioning model (CART) predicting early reperfusion with IV tPA. Each subgroup (rectangle) has the percentage of subjects with early reperfusion ( $95 \%$ confidence interval). The number of subjects reperfused/number of subjects in each subgroup is italicized below each percentage. In all, 33/192 (17.19\%) patients with ICA and MI MCA clots achieved early reperfusion. Tree end points are highlighted in red. Splitting criteria and subgroup characteristics are described along each connecting line.

ual flow (grades $1-2$ versus 0$)$, clot length $(\leq 15 \mathrm{~mm}$ versus $>15 \mathrm{~mm}$ ), cirHU ( $\geq 2$ versus $<2$ ), and distance from the M1 MCA origin to the proximal clot interface $(\leq 10 \mathrm{~mm}$ versus $>10 \mathrm{~mm}$; all patients with ICA occlusions had length imputed to 0$)$.

We restricted the model to include only patients with ICA, tandem, and M1 MCA occlusions. This was deliberate because in this population of patients with proximal occlusions, a decision analysis model helps clinicians decide if early reperfusion with IV tPA is so low that rescue IA therapy may be worthwhile. This part of the analysis was performed by using $R$ statistical computing software, Version 3.0.1 (http://www.r-project.org). The ANOVA method was chosen so that the nodes would be reported as the proportion of recanalization in each subgroup. Early reperfusion rates, including 95\% confidence intervals, were calculated for each of the nodes after the final model was determined. Two readers (S.M.M. and M.E.) assessed all clot characteristics blinded to all follow-up data. Interrater reliability for each clot characteristic and for TICI on the first angiogram of the ipsilesional arterial tree on DSA is reported in 30 patients by using an unweighted $\kappa$ and was interpreted as per the Landis and Koch template.

\section{RESULTS}

We identified 228 patients ( $50.4 \%$ men; median age, 69 years; median baseline NIHSS score, 17) who fulfilled the inclusion criteria. Median symptom onset to IV tPA time was 120 minutes (interquartile range $[\mathrm{IQR}]=70$ minutes); median IV tPA to first angiography time was 70.5 minutes $(\mathrm{IQR}=62$ minutes). Baseline demographics and clot characteristics by center are described in Table 1. Early reperfusion rates with IV tPA, final reperfusion rates after IA therapy, and final clinical outcome are reported in Fig 5.

\section{Primary Analysis}

In analyses defining early reperfusion as TICI $2 \mathrm{a} / 2 \mathrm{~b} / 3$, median clot length in the early reperfusers was $19 \mathrm{~mm}(\mathrm{IQR}=12.9 \mathrm{~mm})$ 


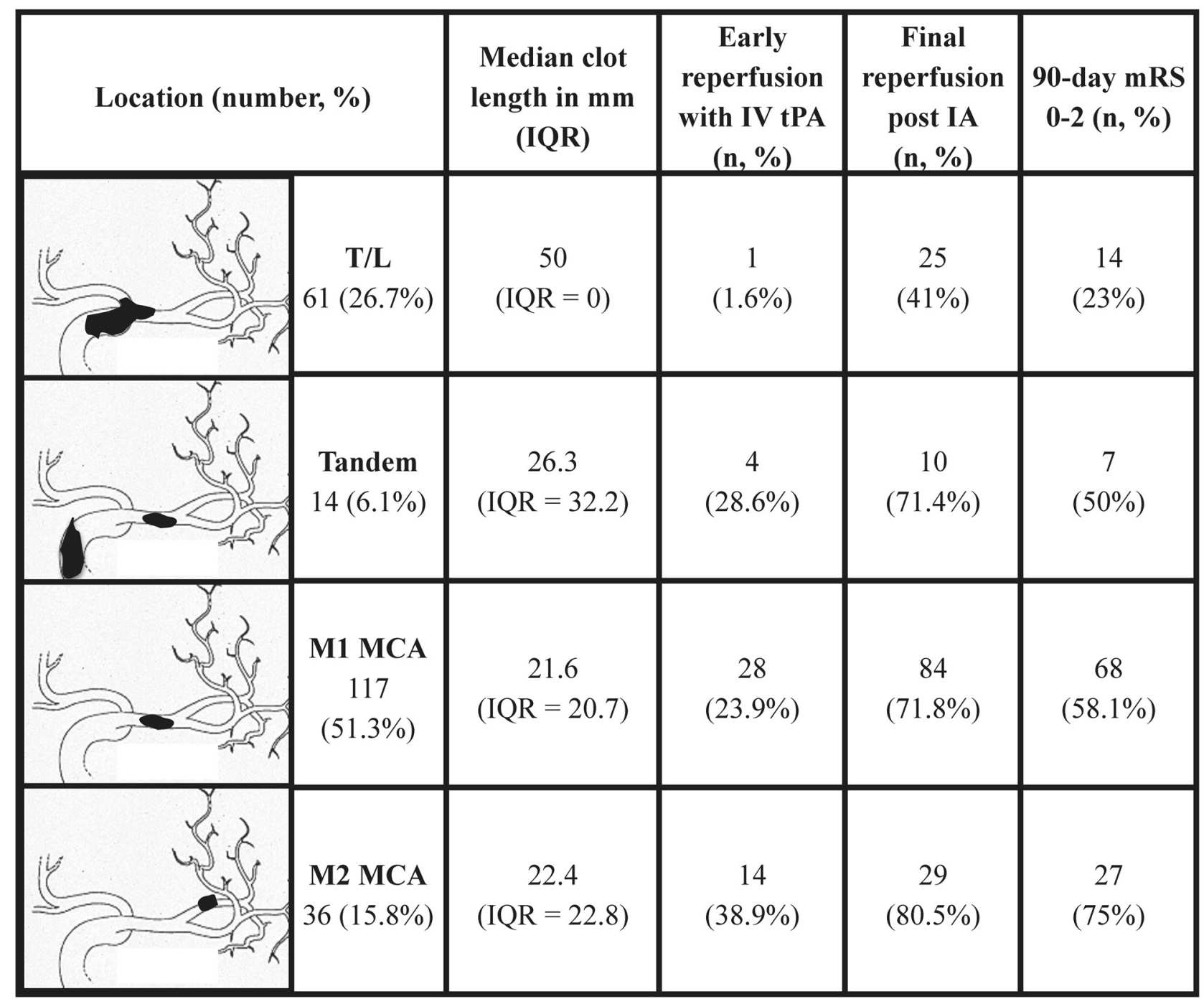

FIG 5. Clot location and length on baseline CTA along with early reperfusion ( $T I C I 2 a / 2 b / 3)$ rates with IV tPA, final reperfusion (TICI $2 b / 3)$ at end of the IA procedure, and 90-day clinical outcome (mRS 0-2).

compared with the nonreperfusers $(34.9 \mathrm{~mm}, \mathrm{IQR}=30.7 \mathrm{~mm}$, $P<.001)$. Residual flow was present in 39/228 (17.1\%) patients. Early reperfusion was seen in $27 / 189(14.3 \%)$ patients without any residual flow (grade 0$)$, in $13 / 30(43.3 \%)$ patients with grade 1 residual flow, and in 7/9 $(77.8 \%)$ patients with grade 2 residual flow $(P<.001)$. CirHU could be measured in 165/228 $(72.4 \%)$ patients. Median cirHU in the early reperfusers was $1.48(\mathrm{IQR}=$ $0.64)$ compared with the nonreperfusers $(1.88, \mathrm{IQR}=1.41, P<$ $.01)$. In patients with a cirHU $<2$, early reperfusion was seen in $37 / 101(36.6 \%)$ compared with $9 / 64(14.1 \%)$ with cirHU $\geq 2$ $(P<.01)$.

\section{Sensitivity Analyses Restricted to M1 MCA Occlusions}

We identified 117 patients with M1 MCA occlusion. Residual flow was present in 28/117 (23.9\%) patients. Early reperfusion (TICI $2 \mathrm{a} / 2 \mathrm{~b} / 3$ ) was seen in $15 / 91(16.5 \%)$ patients without any residual flow at the site of the clot (grade 0), in 10/21 (47.6\%) patients with grade 1 residual flow, and in $3 / 5(60 \%)$ patients with grade 2 residual flow $(P=.002)$. Median clot length in the early reperfusers was $17.1 \mathrm{~mm}(\mathrm{IQR}=14.8 \mathrm{~mm})$ compared with the nonrep- erfusers $(22.7 \mathrm{~mm}, \mathrm{IQR}=19.3, P=.01)$. cirHU could be measured in $116 / 117$ patients. Median cirHU in the early reperfusers was $1.53(\mathrm{IQR}=6.5)$ compared with the nonreperfusers $(2.05$ $\mathrm{mm}, \mathrm{IQR}=1.5, P<.01)$. In patients with a cirHU $<2$, early reperfusion was seen in 23/66 (34.8\%) compared with 5/50 (10\%) patients with cirHU $\geq 2(P=.002)$. Median M1 origin to proximal clot interface distance in the early reperfusers was $13.4 \mathrm{~mm}$ $(\mathrm{IQR}=10.95 \mathrm{~mm})$ compared with $9.3 \mathrm{~mm}(\mathrm{IQR}=7.7 \mathrm{~mm})$ in the nonreperfusers $(P=.001)$. When clots were located $>10 \mathrm{~mm}$ from the M1 MCA origin, 20/60 (33.3\%) showed early reperfusion compared with $8 / 57(14 \%)$ in clots that were $\leq 10 \mathrm{~mm}$ from the M1 MCA origin $(P=.01)$.

\section{Sensitivity Analyses Defining Early Reperfusion as $\mathrm{TICI} 2 \mathrm{~b} / 3$}

Early reperfusion on the first angiography run was seen in $1 / 61$ (1.6\%) of "T/L" type occlusions, in 3/14 (21.4\%) of tandem occlusions, in 14/117 (12\%) of M1 MCA occlusions, and in 5/36 (14\%) of M2 MCA occlusions $(P=.02)$. Early reperfusion (TICI $2 \mathrm{~b} / 3$ ) was seen in $7 / 189(3.7 \%)$ patients without any residual flow 
Table 2: Interrater reliability of various clot characteristics on CTA and TICl on first-run angiography $(n=30)$

\begin{tabular}{lcll}
\hline \multicolumn{1}{c}{ Clot Characteristics } & Agreement & $\boldsymbol{\kappa}$ & $\boldsymbol{P}$ Value \\
\hline Residual flow (grades 0, 1, 2) & $86.7 \%$ & 0.66 & $<.0001$ \\
Clot length $(\leq 15$ vs $>15 \mathrm{~mm})$ & $100 \%$ & 1 & $<.0001$ \\
$\begin{array}{l}\text { Distance from M1 MCA origin } \\
\quad(\leq 10 \text { vs }>10 \mathrm{~mm})\end{array}$ & $100 \%$ & 1 & $<.0001$ \\
$\begin{array}{l}\text { cirHU } \geq 2 \text { vs }<2) \\
\text { Early reperfusion with IV tPA }\end{array}$ & $90 \%$ & 0.78 & $<.0001$ \\
$\quad(\mathrm{TICl} 2 \mathrm{a}-3)$ & $100 \%$ & 1 & $<.0001$ \\
\hline
\end{tabular}

at the site of the clot (grade 0), in 11/30 (36.7\%) patients with grade 1 residual flow, and in 5/9 (55.5\%) patients with grade 2 residual flow $(P<.001)$. Median clot length in the early reperfusers was $14.6 \mathrm{~mm}(\mathrm{IQR}=13.8 \mathrm{~mm})$ compared with the nonreperfusers $(32 \mathrm{~mm}, \mathrm{IQR}=30.9 \mathrm{~mm}, P<.001)$. In patients with a cirHU $<2$, early reperfusion was seen in $21 / 101$ (20.8\%) compared with $1 / 64(1.6 \%)$ patients with cirHU $\geq 2(P<.001)$.

\section{CART Model}

When using recursive partitioning (CART) and restricting the analyses to patients with ICA and M1 occlusions only, residual flow within the clot (grades 1-2) was the most discriminative predictor of early reperfusion (TICI $2 \mathrm{a}-3$ ) followed by clot location, length, distance from M1 origin, and cirHU. In patients with residual flow, 16/34 (47.1\%) reperfused compared with 17/158 $(10.8 \%)$ without residual flow. Among patients with clots having residual flow, those with a shorter clot length $(\leq 15 \mathrm{~mm})$ had a $70.6 \%$ rate of early reperfusion compared those with longer clots $(>15 \mathrm{~mm})$ with $23.5 \%$ early reperfusion. When residual flow was absent, $1.7 \%$ of patients with a carotid-T/-L occlusion reperfused early compared with $16.3 \%$ of patients with tandem or M1 MCA clots. Of patients without residual flow with tandem/M1 occlusions, if the distance of the clot from the M1 MCA origin was $\leq 10$ $\mathrm{mm}, 8 \%$ of patients reperfused early compared with a $25 \%$ early reperfusion rate in patients with clots $>10 \mathrm{~mm}$ from the M1 MCA origin. Last, in those patients with clots $>10 \mathrm{~mm}$ from the M1 MCA origin, early reperfusion was seen in $36.8 \%$ of patients with cirHU $<2$ compared with $17.2 \%$ in patients with cirHU $\geq 2$. The decision tree derived from recursive portioning along with $95 \%$ confidence intervals around estimates of early reperfusion is shown in Fig 4.

Finally, in sensitivity analyses restricted to the Calgary and Keimyung populations, we did not notice any differential effect of clot characteristics on early reperfusion rates (data not shown). Interrater reliability for all clot characteristics and for TICI on the first DSA was substantial (Table 2).

\section{DISCUSSION}

In the largest sample to date, we show that clot characteristics on baseline CTA, including location, length, residual flow, and blood flow around the clot (cirHU), can be used to estimate early reperfusion rates with IV tPA (Fig 4). We also show the generalizability of our results by their applicability in 2 different populations. Our results will inform trialists and physicians of patients who could reperfuse early with IV tPA and those who are more likely to require additional IA therapy to achieve early reperfusion.

Our data show that reperfusion rates with IV tPA are lower when clots are longer. This effect of clot length on lysis has been corroborated by previous studies by using other imaging modalities, including NCCT. ${ }^{3,9}$ Furthermore, by using CART, we are able to show that early reperfusion with IV tPA is highest in clots having residual flow that are, in addition, short. Residual flow within a clot is associated with less tissue damage in coronary angiography and higher rates of arterial recanalization in patients with stroke by using TCD with IV tPA. ${ }^{14,23}$ Residual flow within the clot could be a surrogate for intrinsic clot properties (porous versus impermeable). ${ }^{22,24}$ Our study, for the first time, describes a potential imaging marker on CTA for residual flow within a clot. The lack of any significant association between residual flow on CTA and the hyperattenuated sign on NCCT suggests that residual flow grade was not influenced by intrinsic clot attenuation in our study. ${ }^{24,25}$

In vitro studies show that clot lysis increases if more clot surface is exposed to blood. ${ }^{22}$ Our study uses a novel measure (cirHU) for the extent of blood flow around the clot. CirHU is a marker of good collateral flow and/or residual flow through the clot. It helps in further discrimination of early reperfusion rates (Fig 4). We also confirm a previously reported association between early recanalization and the distance of the clot from the M1 MCA origin. ${ }^{20,21}$

Our study has limitations. We restricted our analysis of early reperfusion rates with IV tPA to baseline CTA characteristics. Nonetheless, no relationship between hyperdense sign on NCCT and residual flow on CTA in our data attests to the fact that these imaging modalities measure very different clot qualities. Moreover, detailed analysis of clot characteristics on NCCT requires thin sections to which we did not have access. ${ }^{3}$ We could not directly measure residual flow within the clot by using another imaging technique like TCD because of the retrospective nature of our study; nonetheless, we show that the imaging marker we propose for residual flow correlates with early reperfusion rates with IV tPA, thus providing a measure of construct validity for our hypothesis. Because we only included patients who were taken to the angiography suite for IA thrombolysis, a selection bias toward patients with larger clots and more severe ischemia is possible in our sample. Finally, our sample includes patients from 2 different centers, one of which is predominantly East Asian. This latter population is known to have higher rates of intracranial atherosclerosis. Besides, differences in CT scanning equipment and acquisition protocol (autotriggering) between the 2 centers could explain the differences in some baseline CTA imaging measures (Table 1). Nonetheless by including data from both centers, we are able to increase our sample size and the generalizability of our findings. ${ }^{13}$

\section{CONCLUSIONS}

Our results and the decision analysis (CART) algorithm we describe in Fig 4 need to be validated in a larger prospective cohort of patients. Such a predictive model of early reperfusion by using baseline imaging could help clinical decision-making when administering IV tPA and IA therapy in addition to being used in the targeted design of clinical trials in patients with acute ischemic stroke. 
Disclosures: Mayank Goyal—UNRELATED: Consultancy: Covidien/ev3, Comments: for design, conducting, and educating related to acute stroke trials; Payment for Lectures (including service on Speakers Bureaus): Covidien/ev3, Comments: education on acute stroke imaging and treatment; Stock/Stock Options: Calgary Scientific, NoNo Inc, Comments: own stock. Andrew M. Demchuk-RELATED: The Calgary CTA database was supported by Heart and Stroke Foundation of Alberta, North West Territories and Nunavut funding, and the Heart and Stroke Foundation Chair in Stroke Research. * UNRELATED: Grants/Grants Pending: receives grant support from the Canadian Institute of Health Research to study clot characteristics predicting early reperfusion with IV tPA. * Bijoy K. Menon-UNRELATED: Grants/Grants Pending: Canadian Institute for Health Research, ${ }^{*}$ Comments: grant for a prospective study aimed at understanding reperfusion with IV tPA. This project, however, does not use any data from the above-mentioned study. ${ }^{\star}$ Money paid to the institution.

\section{REFERENCES}

1. von Kummer R, Meyding-Lamade U, Forsting M, et al. Sensitivity and prognostic value of early CT in occlusion of the middle cerebral artery trunk. AJNR Am J Neuroradiol 1994;15:9-15

2. Leys D, Pruvo JP, Godefroy O, et al. Prevalence and significance of hyperdense middle cerebral artery in acute stroke. Stroke 1992; 23:317-24

3. Riedel CH, Jensen U, Rohr A, et al. Assessment of thrombus in acute middle cerebral artery occlusion using thin-slice nonenhanced computed tomography reconstructions. Stroke 2010;41:1659-64

4. Moulin T, Cattin F, Crepin-Leblond T, et al. Early CT signs in acute middle cerebral artery infarction: predictive value for subsequent infarct locations and outcome. Neurology 1996;47:366-75

5. Tomsick T, Brott T, Barsan W, et al. Prognostic value of the hyperdense middle cerebral artery sign and stroke scale score before ultraearly thrombolytic therapy. AJNR Am J Neuroradiol 1996;17: $79-85$

6. Somford DM, Nederkoorn PJ, Rutgers DR, et al. Proximal and distal hyperattenuating middle cerebral artery signs at CT: different prognostic implications. Radiology 2002;223:667-71

7. Leary MC, Kidwell CS, Villablanca JP, et al. Validation of computed tomographic middle cerebral artery "dot" sign: an angiographic correlation study. Stroke 2003;34:2636-40

8. Barber PA, Demchuk AM, Hudon ME, et al. Hyperdense Sylvian fissure MCA “dot” sign: a CT marker of acute ischemia. Stroke 2001;32:84-88

9. Riedel CH, Zimmermann P, Jensen-Kondering U, et al. The importance of size: successful recanalization by intravenous thrombolysis in acute anterior stroke depends on thrombus length. Stroke 2011;42:1775-77

10. Shobha N, Bal S, Boyko M, et al. Measurement of length of hyperdense MCA sign in acute ischemic stroke predicts disappearance after IV tPA. J Neuroimaging 2014;24:7-10

11. Bhatia R, Hill MD, Shobha N, et al. Low rates of acute recanalization with intravenous recombinant tissue plasminogen activator in ischemic stroke: real-world experience and a call for action. Stroke 2010;41:2254-58

12. Puetz V, Dzialowski I, Hill MD, et al. Intracranial thrombus extent predicts clinical outcome, final infarct size and hemorrhagic transformation in ischemic stroke: the clot burden score. Int J Stroke 2008;3:230-36

13. Lee KY, Han SW, Kim SH, et al. Early recanalization after intravenous administration of recombinant tissue plasminogen activator as assessed by pre- and post-thrombolytic angiography in acute ischemic stroke patients. Stroke 2007;38:192-93

14. Saqqur M, Tsivgoulis G, Molina CA, et al. Residual flow at the site of intracranial occlusion on transcranial Doppler predicts response to intravenous thrombolysis: a multi-center study. Cerebrovasc Dis 2009;27:5-12

15. Ma M, Berger J. A novel TCD grading system for residual flow in stroke patients. Stroke 2001;32:2446

16. Rha JH, Saver JL. The impact of recanalization on ischemic stroke outcome: a meta-analysis. Stroke 2007;38:967-73

17. Wintermark M, Albers GW, Broderick JP, et al. Acute stroke imaging research roadmap II. Stroke 2013;44:2628-39

18. Lemon SC, Roy J, Clark MA, et al. Classification and regression tree analysis in public health: methodological review and comparison with logistic regression. Ann Behav Med 2003;26:172-81

19. Menon BK, Smith EE, Coutts SB, et al. Leptomeningeal collaterals are associated with modifiable metabolic risk factors. Ann Neurol 2013;74:241-48

20. Hirano T, Sasaki M, Mori E, et al. Residual vessel length on magnetic resonance angiography identifies poor responders to alteplase in acute middle cerebral artery occlusion patients: exploratory analysis of the Japan Alteplase Clinical Trial II. Stroke 2010;41:2828-33

21. Saarinen JT, Sillanpaa N, Rusanen H, et al. The mid-M1 segment of the middle cerebral artery is a cutoff clot location for good outcome in intravenous thrombolysis. Eur J Neurol 2012;19:1121-27

22. Anand M, Rajagopal K, Rajagopal KR. A model for the formation and lysis of blood clots. Pathophysiol Haemost Thromb 2005;34: 109-20

23. Blanke $H$, Cohen $M$, Karsch KR, et al. Prevalence and significance of residual flow to the infarct zone during the acute phase of myocardial infarction. J Am Coll Cardiol 1985;5:827-31

24. Liebeskind DS, Sanossian N, Yong WH, et al. CT and MRI early vessel signs reflect clot composition in acute stroke. Stroke 2011;42: 1237-43

25. Moftakhar P, English JD, Cooke DL, et al. Density of thrombus on admission CT predicts revascularization efficacy in large vessel occlusion acute ischemic stroke. Stroke 2013;44:243-45 